\title{
Multi-source fault detection and diagnosis based on multi-level Knowledge Graph and Bayesian theory reasoning
}

\author{
Tao Sun \\ School of Computer Science and Technology \\ Qilu University of Technology (Shandong Academy of \\ Sciences) \\ Jinan, China \\ suntao0906@163.com
}

\author{
Qi Wang \\ School of Computer Science and Technology \\ Qilu University of Technology (Shandong Academy of \\ Sciences) \\ Jinan, China \\ yining1104@sina.com
}

\begin{abstract}
In complex industrial processes, complex associations are often involved. This complex relationship makes traditional fault detection and diagnosis methods difficult to achieve satisfactory results in multi-source fault detection and diagnosis. Therefore, this paper proposes a new multi-source fault detection and diagnosis framework. The method can successfully detect the state of the system, and at the same time, can locate the fault source simply and quickly. This method firstly constructs the multi-level knowledge graph in the complex industrial process, and then use the discriminant coefficient $R$ to detect whether the system has failed. If the system fails into the fault diagnosis stage, the probability of the fault is derived based on Bayesian theory. This article describes the framework in detail. The TE process is taken as an example to prove the effectiveness of the method.
\end{abstract}

Keywords-complex industrial process, knowledge graph, bayesian theory, fault detection and diagnosis

\section{INTRODUCTION}

With the development of computer technology and the promotion of concepts such as Industry 4.0, modern industrial processes tend to be more automated, integrated, complex and intelligent. In the actual process monitoring, due to the large scale of industrial processes and complex business logic, there is a complex relationship between process variables in the production process. At the same time, with the increasing complexity of the process, the influencing factors are gradually increasing, and multiple faults occur frequently in complex industrial systems. However, the traditional fault diagnosis technology is mostly carried out under the condition of single fault type and simple influencing factors, and its accuracy is greatly reduced in the face of complex industrial processes. Many factors make the fault diagnosis of complex industrial system more and more difficult.

How to construct and mine the relationship in complex industrial systems and use these associations to improve the accuracy of fault detection and diagnosis is a key issue that needs to be studied at present. The Knowledge Graph (KG) [1] can describe the various entities and concepts that exist in the real world and the relationships between these entities and concepts. Based on the concept map of knowledge graph, mining the associations in complex industrial systems and displaying them in the form of graphs can more intuitively present this intricate relationship. At the same time, it can describe the abstract concepts of different levels and granularities, and integrate the larger resources in complex industrial systems. The fault source is located by inferring the constructed knowledge graph. Therefore, this paper proposes a new fault detection and diagnosis framework for many

DOI reference number: 10.18293/SEKE2019-064

This work was supported in part by Shandong Natural Resources Fund (NO.02053522), Shandong Province Graduate Education Innovation Program (NO.24170404), and Qilu University of Technology Teaching and Research Project (NO.041201034109). problems in complex industrial systems, which combines knowledge graph and bayesian inference, and conducts experiments on TE data sets to verify its effectiveness.

\section{RELATED WORK}

Once an accident occurs in a complex industrial process, it may cause serious adverse effects on production safety, efficiency or product quality. At present, the widely used methods in fault detection and diagnosis (FDD) of complex industrial processes include: analytical mathematical modelbased method, knowledge-based method, data-driven method, etc.

The analytical model-based approach [2-4] has great limitations in application due to the need to establish accurate mathematical models. Knowledge-based methods are mainly divided into two types: causal graph method [5-7] and fault tree method [8]. Zhang $\mathrm{K}$ et al. [5] proposed a kernel-based conditional independence test for conditional independence testing in Bayesian network learning and causal discovery. Experimental results show that it is superior to other methods. Caceres et al. [8] proposed to establish the fault tree according to the structural block diagram of the system. However, the defect of knowledge-based approach is that it relies too much on production experience and process knowledge. The datadriven FDD method [9-10] achieves fault detection and diagnosis of the system by analyzing and processing a large number of process data including system normal and fault information. However, the diagnostic accuracy of data-driven methods often depends heavily on the completeness and representativeness of the fault samples.

Both knowledge-based and data-driven approaches have their own shortcomings. Therefore, this paper proposes a method for fault detection and diagnosis combining knowledge-based methods with data-driven methods. Knowledge graph [1] is an emerging research field in recent years. It can express more information than traditional knowledge-based methods. This method is mainly used in natural language processing, medical[11], financial and other fields. However, the application of this method in complex industrial fields is rare. Knowledge graph is a graph model in essence, and fault diagnosis based on knowledge graph can be solved by graph model theory. However, Bayesian network $(\mathrm{BN})$ is a typical probability graph model, which, considering the network structure and node attribute information, has a solid theoretical basis of probability theory and is widely used. It is suitable for expressing and analyzing uncertain knowledge and can make effective inference to uncertain knowledge. Therefore, this paper uses the combination of knowledge graph and Bayesian theory to detect and diagnose faults in complex industrial systems. 


\section{Fault Detection AND Diagnosis Method Based ON MULTI-LEVEL KNOWLEDGE GRAPH}

In complex industrial systems, there are many levels of factors that affect the state of production. For example, production level, process level, energy saving and emission reduction level, and raw material level. Therefore, the framework proposed in this paper analyzes complex industrial systems from multiple levels and constructs a multi-level knowledge graph, and then makes inferential diagnosis and fault diagnosis based on this knowledge graph. The flowchart of the framework is shown in Figure 1.

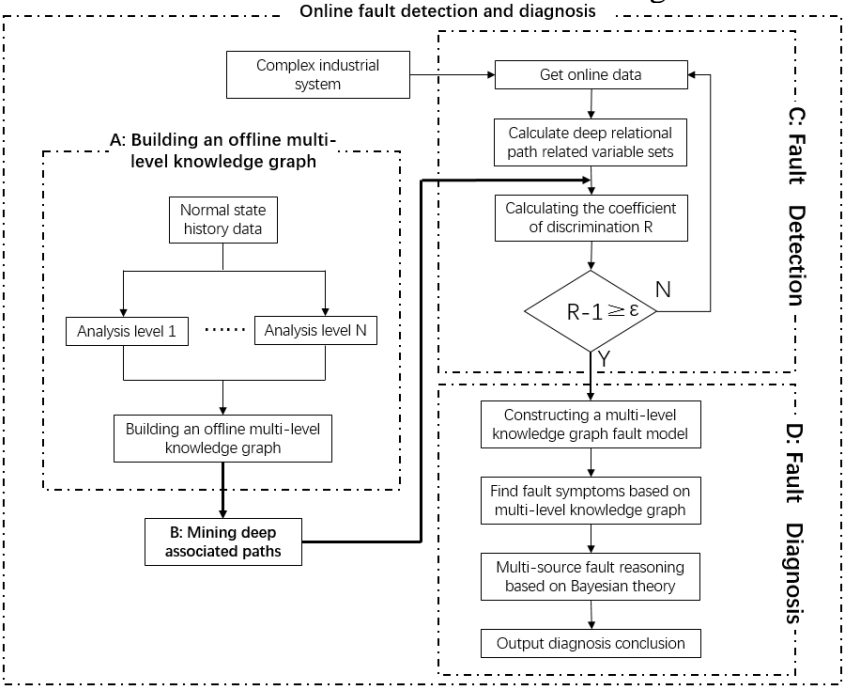

Fig. 1. Schematic diagram of fault detection and diagnosis model based on multi-level knowledge graph

\section{A. Building an offline multi-level knowledge graph}

The flow of constructing the knowledge graph in Part A of Figure 1 is shown in Figure 2.

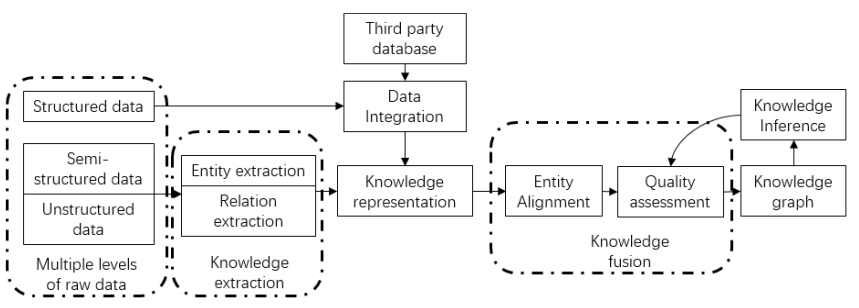

Fig. 2. Schematic diagram of constructing a knowledge graph

There are many differences between the knowledge graph construction of complex industrial systems and the construction of general knowledge graph. Data sources in complex industrial systems come not only from the Internet, but also from all levels affecting production. If the knowledge graph is not comprehensive enough, the time for fault detection and diagnosis will become longer and the accuracy will decrease. First, collect data from multiple levels in a complex industrial system, and then extract the knowledge from the original data. Because the relationship between complex industrial system entities is particularly complex, this paper focuses on the relationship extraction in knowledge extraction. In this paper, Pearson correlation coefficient method is used to find the correlation between entities (the relationship between moderate correlation and high correlation is adopted [12]), and at the same time, prior knowledge is combined to extract the relationship. The extracted knowledge elements are then represented for further processing. Then there is data fusion, the purpose of which is to fuse the knowledge acquired by different data sources to construct the association between the data. Knowledge graph are constructed by merging knowledge through entity alignment (judging whether two entities match) and quality assessment (inconsistency verification: identifying potential contradictions through rules). Figure 3 is a schematic diagram of the knowledge graph at four levels.

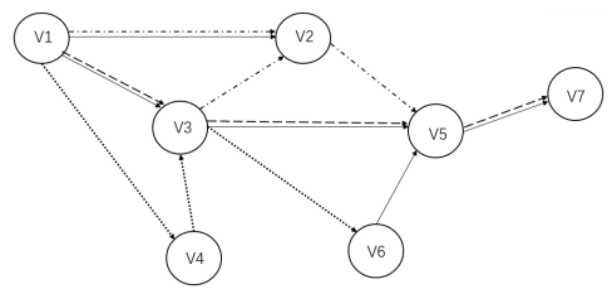

Fig. 3. Schematic diagram of multi-level knowledge graph

\section{B. Mining deep-level associations in multi-level knowledge graphs}

The method of mining deep-level associations in multilevel knowledge graphs in Part B of Figure 1 is as follows: According to the existing relationship in the multi-level knowledge graph, find the variable correlation coefficient of each variable in the knowledge graph as the weight coefficient of the variable, and select a critical path according to the size of the weight coefficient. The variables in this path must appear in the knowledge graph of each level at the same time, so that they can represent the deep relationship of the multilevel knowledge graph. Through the deep relationship in the knowledge graph, it is possible to quickly and conveniently detect whether the system has failed without considering the complex relationship in the entire knowledge graph.

\section{Fault detection method based on multi-level knowledge graph}

The part $\mathrm{C}$ fault detection method in Figure 1 is as follows: The knowledge graph is mainly composed of several triples, that is, pairs of variable correlation coefficients. After the system fails, the correlation coefficient of the variables in the original knowledge graph will inevitably change, that is, the entity changes, and the relationship (weight) between the entities changes. Therefore, this paper uses the changes in these two aspects of the knowledge graph structure to define the discriminant coefficient $\mathrm{R}$ rule. The discriminant coefficient $\mathrm{R}$ rule is defined as follows:

Suppose that $\mathrm{T}$ is used to represent the variable in the deep associated path in the knowledge graph under normal conditions, $\mathrm{T}=\left[\mathrm{T}_{1}, \mathrm{~T}_{2}, \mathrm{~T}_{3} \ldots\right], \mathrm{N}_{\mathrm{i}}$ represents the set of the $\mathrm{i}$-th variable entity pair in $\mathrm{T}, \mathrm{n}$ represents the number of pairs of entities in $\mathrm{N}_{\mathrm{i}} . \mathrm{W}_{\mathrm{i}}$ represents the relationship weight of the $\mathrm{i}$-th pair of entities in N. Similarly, $\mathrm{T}^{\prime}, \mathrm{N}_{\mathrm{i}}^{\prime}$ and $\mathrm{W}_{\mathrm{i}}^{\prime}$ are used to represent the variables under test, and $\mathrm{m}$ is the number of entity pairs in $\mathrm{N}_{\mathrm{i}}^{\prime}$. The calculation rules for the discriminant coefficient $\mathrm{R}$ are as follows:

Let the number of the variable entity pairs in the normal state $T$ and the variable entity pairs in the state $T^{\prime}$ to be detected be the same as $t$, and the set of different entity pairs in the $T$ state and $T^{\prime}$ is $T_{k}$, and the pair of different entities in the $T^{\prime}$ state and $T$ is $T_{k}^{\prime}$. When $T_{k}, T_{k}^{\prime}$ have different entity pairs, $\mathrm{T}_{\mathrm{k}}, \mathrm{T}_{\mathrm{k}}^{\prime}$ are automatically incremented by one. When $\mathrm{T}_{\mathrm{k}}$, $\mathrm{T}_{\mathrm{k}}^{\prime}$ have different entity pairs, $\mathrm{W}_{\mathrm{i}}^{\prime}$ is automatically incremented by 1 . When $T_{k}, T_{k}^{\prime}$ have the same entity pair, and W's relationship weight is inconsistent with the corresponding 
relationship weight in $\mathrm{W}^{\prime} \mathrm{i}, \mathrm{W}^{\prime}$ is automatically incremented by one. The mathematical model of the discriminant coefficient $\mathrm{R}$ is:

$$
R=\frac{\sum T_{k}+\sum T^{\prime} k_{k}+t}{n}+W^{\prime}=\frac{|m-t|+|n-t|+t}{n}+W^{\prime}=\frac{m+n-t}{n}+W^{\prime}
$$

On the basis of an acceptable fault tolerance rate $\varepsilon$, if R-1 $\geq \varepsilon$ is satisfied, it indicates a system failure, otherwise it indicates a normal state.

\section{Fault Diagnosis Method Based On Multi-Level Knowledge Graph}

The D part fault diagnosis method in Figure 1 is as follows:

1) According to the determined fault variables, the moderately and highly correlated variable pairs of correlation coefficients are selected in the knowledge graph relationship pairs of each level to construct a multi-level knowledge graph fault model.

2) According to the fault symptoms of the system, find all possible candidate failure causes. For each candidate failure reason, the posterior probability value under the known fault symptom condition is calculated based on Bayesian theory.

3) Set a certain threshold, and consider the cause of the failure exceeding the threshold as the most likely cause of the failure.

The posterior probability calculation method is as follows:

1) Calculate the first-order cut set expression for each fault symptom $E_{i}$.

$$
\begin{gathered}
E_{i}=e_{t, i} r_{t} \cup e_{j, i} E_{i} \\
E_{j}=e_{k, j} r_{k}
\end{gathered}
$$

Where, $E_{i}$ and $E_{j}$ are fault symptoms, $r_{t}$ and $r_{k}$ are candidate fault causes, and $e_{j, i}$ is the edge from $E_{j}$ to $E_{i} \cdot e_{t, i}$ is the edge from candidate failure cause $r_{t}$ to failure symptom $E_{i}$.

2) Calculate the final cut set expression for each fault symptom $E_{i}$ :

$$
E_{i}=e_{t, i} r_{t} \cup e_{j, i} e_{k, j} r_{k}
$$

The first-order cut set expression is expanded according to the relevant edge direction logic. This process can eliminate all the variable nodes in the fault model and obtain the final cut set expression consisting only of the cause node and the relevant edge.

3) Calculate the posterior probability value $P\left(r_{i} \mid E\right)$ of the fault cause $r_{i}$ : Logging the fault symptoms according to (5) for all fault symptoms, and inserting the corresponding fault cause occurrence probability value and the associated edge probability value to obtain the posterior probability value $\mathrm{P}\left(\mathrm{r}_{\mathrm{i}} \mid \mathrm{E}\right)$ of the final detected fault cause $\mathrm{r}_{\mathrm{i}}$.

$$
\mathrm{P}\left(r_{i} \mid \mathrm{E}\right)=\frac{P\left(r_{i}, E_{1}, \ldots E_{n}\right)}{P\left(E_{1}, \ldots E_{n}\right)}=\frac{P\left(r_{i} \cap E_{i} \ldots E_{n}\right)}{P\left(E_{1} \cap \ldots E_{n}\right)}
$$

Where, $\mathrm{E}=(\mathrm{E}=\mathrm{E} 1, \ldots \mathrm{En})$ is the set of fault symptoms, $\mathrm{n}$ is the number of abnormal variables, and $r_{i}$ is the cause of the fault.

\section{EXPERIMENT}

\section{A. Data Sets}

The data set used in this paper is the data of TE process, a simulation system of chemical process. For a detailed description of the process, refer to the relevant literature[13]. The 22 process measurement variables in the TE model are represented by V1, V2...V22 and the 20 failure types are r41, r42...r60.

\section{B. Building a multi-level knowledge graph of the TE process}

Limited by the TE process simulation data, this paper constructs the TE process knowledge graph from two levels, as shown in Figure 4. The solid line represents the technological process level, and the dotted line represents the data level of the production process.

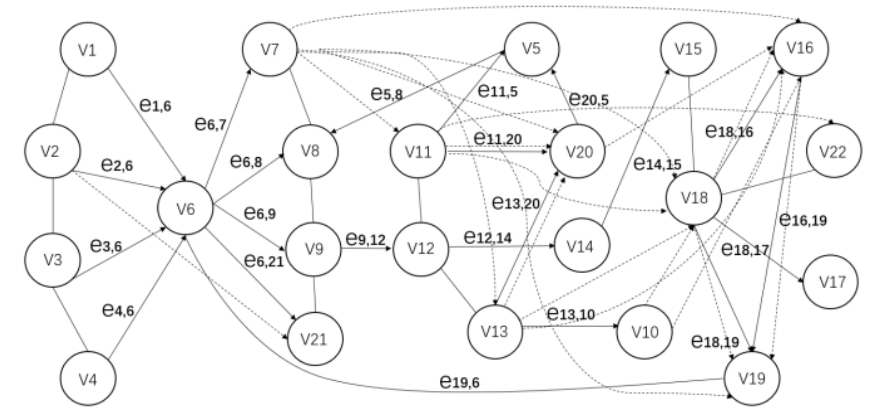

Fig. 4. Schematic diagram of the multi-level knowledge graph of the TE process

\section{Mining deep-level associations in multi-level knowledge graph of TE processes}

First, the weighting coefficients of each successive measured variable are analyzed according to the constructed multi-level knowledge graph, The weights corresponding to the measured variables $\{\mathrm{V} 2, \mathrm{~V} 7, \mathrm{~V} 10, \mathrm{~V} 11, \mathrm{~V} 13, \mathrm{~V} 16, \mathrm{~V} 18$, $\mathrm{V} 19, \mathrm{~V} 20\}$ are $\{1,6,2,3,4,5,7,2,4\}$.

According to the TE process and the principle of large weight coefficient, the deep correlation path of the multi-level knowledge graph can be obtained as follows: V2, V7, V13, V16, V18, V20. Analysis of the four variables of the TE process feed shows that the V2 variable is highly correlated with other variables. This determines that V2 is the starting variable of the deep associated path. This path represents the deep interrelationship of the equipment (feed-reactorseparator-compressor-stripper).

\section{TE process fault detection method based on multi-level knowledge graph}

In order to prove the validity of the method, this experiment selected single source fault (fault 7), multi-source fault: fault $(5,7)$ and fault $(2,6,13)$.

According to the fault detection method proposed above, the discrimination coefficient $\mathrm{R}$ in the four production states is calculated. It is judged whether the TE chemical system is in a fault state according to the change of the discrimination coefficient $\mathrm{R}$. The parameters $\mathrm{R}$ in the four production states are shown in Figure 5. It can be seen from Figure 5 that the discriminant coefficient $\mathrm{R}$ completely separates the data of the normal state and the fault state, and accurately detects the system fault. 


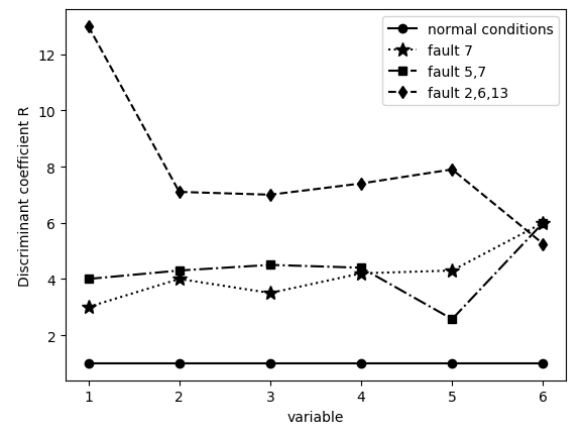

Fig. 5. Schematic diagram of the comparison between the normal state and the fault state discrimination coefficient $\mathrm{R}$

\section{E. TE process fault diagnosis method based on multi-level knowledge graph}

In this experiment, fault 5 and fault 7 were selected as the multi-source fault data set for experiment. The specific diagnostic steps are as follows:

1) Building a knowledge graph failure model in the current state as shown in Figure 6.

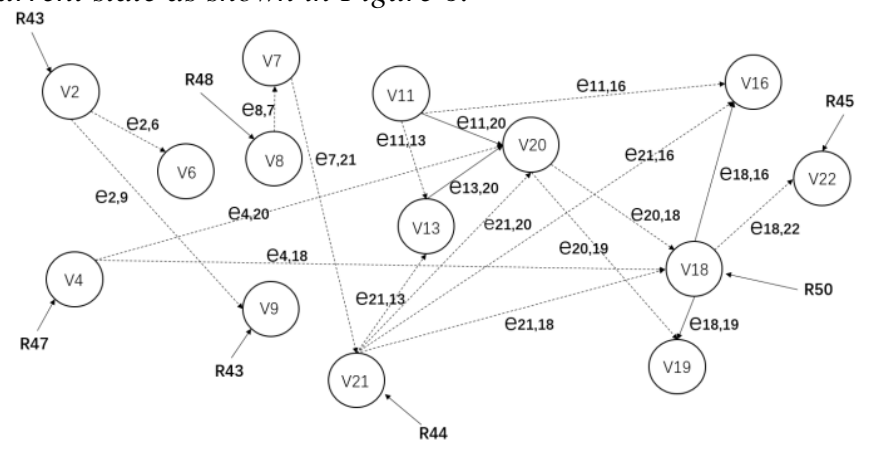

Fig. 6. Schematic diagram of multi-level knowledge graph failure model

2) Calculate the posterior probability value of each candidate failure cause.

According to (2)-(5), the diagnosis results are shown in Table 1. It can be seen from the table that the posterior probability of $r_{45}$ and $r_{47}$ is significantly larger than $r_{43}, r_{44}, r_{48}$, $r_{50}$. Therefore, the cause node $r_{45}$ (condenser cooling water feed temperature) and $\mathbf{r}_{47}$ (flow rate $4 \mathrm{C}$ pressure loss) are the cause of the failure, and the diagnosis conclusion is consistent with the original assumption.

TABLE I.

DiAGNOSTIC RESULTS OF THE CAUSE NODE

\begin{tabular}{|c|c|}
\hline Candidate failure cause & $\begin{array}{c}\text { Percentage of } \\
\text { posterior probability }\end{array}$ \\
\hline r43:D temperature in flow 2 & $14.11 \%$ \\
\hline r44: Reactor cooling water feed temperature & $7.29 \%$ \\
\hline r45: condenser cooling water feed temperature & $65.47 \%$ \\
\hline r48: Changes in A, B, and C components in Flow 4 & $50.32 \%$ \\
\hline r50: C material temperature in flow 4 & $10.51 \%$ \\
\hline
\end{tabular}

TABLE II. DIAGNOSTIC TIME COMPARISON

\begin{tabular}{|c|c|}
\hline Diagnosis method & Diagnostic time \\
\hline PCA_KNN & 0.446 \\
\hline PCA_SVM & 0.413 \\
\hline PNN & 0.375 \\
\hline KG_Bayesian network & 0.306 \\
\hline
\end{tabular}

Table 2 compares the diagnostic time of the four methods of PCA_KNN, PCA_SVM, PNN, KG_Bayesian network. It can be seen that the method used in this paper takes less time than the traditional fault detection and diagnosis methods.

\section{CONCLUSION}

Based on the strong correlation between knowledge graphs and comprehensive consideration of various factors, this paper constructs a multi-level knowledge graph in complex industrial processes. This way of building knowledge graphs maximizes the consideration of influencing factors in complex industrial systems. By mining the correlation law between variables, the discriminant coefficient method is used to detect the state of the system. The method has achieved good results in the TE process and can accurately identify the system state. The multi-level knowledge graph fault diagnosis method based on Bayesian theory provides a new idea for fault diagnosis of complex industrial systems. This method has achieved good results in reasoning the multi-source failure of complex industrial systems. At the same time, this method can diagnose the fault source easily and quickly. At present, this method is only applicable to the processing of existing fault cause data, and the next step will be to study the fault selflearning function.

\section{REFERENCES}

[1] Huang Z , Chung W , Ong T H , et al. [ACM Press the second ACM/IEEE-CS joint conference - Portland, Oregon, USA (2002.07.142002.07.18)] Proceedings of the second ACM/IEEE-CS joint conference on Digital libraries, - JCDL I"02 - A graph-based recommender system for digital library[J]. 2002:65

[2] Kinnaert, Michel. Fault diagnosis based on analytical models for linear and nonlinear systems - a tutorial[J]. IFAC Proceedings Volumes, 2003 , 36(5):37-50

[3] Liao Z, Wen F, Guo W, et al. An analytic model and optimization technique based methods for fault diagnosis in power systems[C]// International Conference on Electric Utility Deregulation \& Restructuring \& Power Technologies. IEEE, 2008.

[4] Cui Y, Shi J , Wang Z . An analytical model of electronic fault diagnosis on extension of the dependency theory[J]. Reliability Engineering \& System Safety, 2015, 133:192-202.

[5] Zhang K, Peters J, Janzing D, et al. Kernel-based conditional independence test and application in causal discovery $[\mathrm{J}]$. arXiv preprint arXiv:1202.3775, 2012

[6] Iri M , Aoki K, O"Shima E, et al. An algorithm for diagnosis of system failures in the chemical process[J]. Computers \& Chemical Engineering, 1979, 3(1-4):489-493

[7] Yang F , Sirish L S , Xiao D . Signed Directed Graph modeling of industrial processes and their validation by data-based methods[C]// Control \& Fault-tolerant Systems. IEEE, 2010

[8] Caceres S , Henley E J . Process Failure Analysis by Block Diagrams and Fault Trees[J]. Industrial \& Engineering Chemistry Research, 1976, 15(2):128-134

[9] Zhang J, Zhu Y, Shi W, et al. An Improved Machine Learning Scheme for Data-Driven Fault Diagnosis of Power Grid Equipment[C]// IEEE International Conference on High Performance Computing \& Communications. IEEE, 2015

[10] Wang D , Man Z . Special issue: Data-driven fault diagnosis of industrial systems[J]. Information Sciences, 2014, 259:231-233

[11] Fang Y, Wang H, Wang L, et al. Diagnosis of COPD Based on a Knowledge Graph and Integrated Model[J]. IEEE Access, 2019, 7: 46004-46013

[12] Kim Y, Kim T H, Ergün T. The instability of the Pearson correlation coefficient in the presence of coincidental outliers[J]. Finance Research Letters, 2015, 13: 243-257

[13] Chiang L H, Russell E L, Braatz R D. Fault detection and diagnosis in industrial systems[J]. 2001. 